\title{
Critical Behavior of the Hall Conductivity at the Metal-Insulator Transition
}

\author{
Stuart B. Field and T. F. Rosenbaum \\ The James Franck Institute and Department of Physics, University of Chicago, Chicago, Illinois 60637 \\ (Received 6 May 1985)
}

\begin{abstract}
We measure the low-temperature longitudinal and Hall conductivities in a series of Ge:Sb samples at the approach to the metal-insulator transition. Both conductivities critically vanish with the same exponent of 1 , in contradiction to the ratio of 2 predicted by the scaling theory of localization.
\end{abstract}

PACS numbers: $72.15 . \mathrm{Gd}, 71.30 .+\mathrm{h}, 71.55 . \mathrm{Jv}$

The theoretical basis for the continuous nature of the zero-temperature metal-insulator (MI) transition in disordered systems was provided by Abrahams et al., ${ }^{1}$ in 1979. They used scaling arguments to show that, near the critical density $n_{c}$, the conductivity depends on the density $n$ as $\sigma=\sigma_{0}\left[\left(n-n_{c}\right) / n_{c}\right]^{\nu}$, with perturbative methods giving $\nu=1$. The first lowtemperature experimental test ${ }^{2}$ of the theory found $\nu=\frac{1}{2}$, not 1 , in uncompensated Si:P, possibly because of the importance of electron interactions which are not considered in the localization scaling theory. Subsequent experiments on amorphous ${ }^{3} \mathrm{Si}: \mathrm{Nb}$, compensated ${ }^{4} \mathrm{Ge}: \mathrm{Sb}$, and the magnetic semiconductor ${ }^{5} \mathrm{Gd}_{3} \mathrm{~S}_{4}$ gave $\nu=1$. In these systems, Anderson localization might dominate Coulomb effects, and hence the Abrahams et al. approach would be appropriate. Alternatively, a theory ${ }^{6,7}$ considering electron interactions alone in the weak disorder limit might account for the exponent of 1 .

In an extension of the work of Abrahams et al., Shapiro and Abrahams ${ }^{8}$ developed a localization scaling theory for the Hall effect at the MI transition. They found that the exponent $\nu_{\mathrm{H}}$ of the Hall conductivity $\sigma_{\mathrm{H}}$ should be twice that of the ordinary conductivity, $\sigma_{\mathrm{H}}=\sigma_{1}\left[\left(n-n_{c}\right) / n_{c}\right]^{2 \nu}$. Thus, comparing the critical behavior of the ordinary and Hall conductivities should help discern which, if any, theories of the MI transition apply to particular physical systems. In this Letter, we present magnetotransport measurements of $\mathrm{Ge}: \mathrm{Sb}$ at millikelvin temperatures which establish that $\sigma$ and $\sigma_{\mathrm{H}}$ critically vanish at the MI transition with the same exponent, $\nu=\nu_{\mathrm{H}}=1$. This result casts doubt on whether systems with $\nu=1$ are in fact correctly described by the physics of the scaling theory of localization. Unfortunately, no predictions for $\nu_{\mathrm{H}}$ exist at present in the models for an interaction-driven MI transition.

We sliced a series of $\mathrm{Ge}: \mathrm{Sb}$ samples of typical dimensions $9 \times 1 \times 0.4 \mathrm{~mm}^{3}$ from Czochralski-grown single-crystal boules and etched them in CP-4 to remove any damaged surface layer. They were cooled in a top-loading dilution refrigerator where temperatures $T$ below $50 \mathrm{mK}$ were calibrated by the anisotropy of cobalt- 60 decays. A conventional five-probe technique allowed the determination of both the longitudinal and transverse (Hall) voltages. We elucidated the absolute $\mathrm{Sb}$ concentration via Hall effect at $T=300 \mathrm{~K}$, and we established the relative density scale more accurately through the ratio of sample resistances at liquidhelium and room temperatures. ${ }^{9}$ This resistance-ratio technique eliminates uncertainties arising from sample geometry. The Ge:Sb samples were not intentionally compensated. ${ }^{10} \mathrm{~A}$ method to determine the compensation $K$ is demonstrated by Thomas et al., ${ }^{4}$ who found that the steepness of the MI transition (i.e., the prefactor $\sigma_{0}$ ) in compensated $\mathrm{Ge}: \mathrm{Sb}$ decreased as $K$ increased. Comparison of our prefactor $\sigma_{0}$ with those of Thomas et al. ${ }^{4}$ gives $K \sim 20 \%$. We note, however, that it is notoriously difficult to accurately determine $K$ for highly doped semiconductors.

We plot in Fig. 1 the $T=8 \mathrm{mK}$ longitudinal conductivity $\sigma$ as a function of donor density $n$ at a magnetic field $H=2.7 \mathrm{kOe}$. The different symbols correspond to samples cut from different boules. Extrapolations of $8 \mathrm{mK}<T<80 \mathrm{mK}$ data to $T=0$ give corrections of less than $1 \%$. The conductivity follows the characteristic scaling behavior with exponent $\nu=0.9 \pm 0.1$. The solid line is a nonlinear least-squares fit with $\sigma_{0}=3.8 \sigma_{\mathrm{M}}, n_{c}=1.68 \times 10^{17} \mathrm{~cm}^{-3}$, and $\nu=0.90$, where

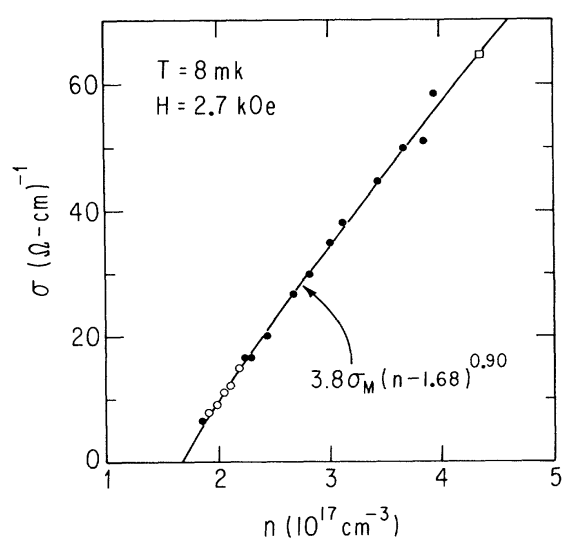

FIG. 1. The low-temperature longitudinal conductivity $\sigma$ vs donor density $n$ at the approach to the MI transition in $\mathrm{Ge}: \mathrm{Sb}$. The solid line is a best fit to the critical form indicated. Different symbols denote samples cut from different boules. 


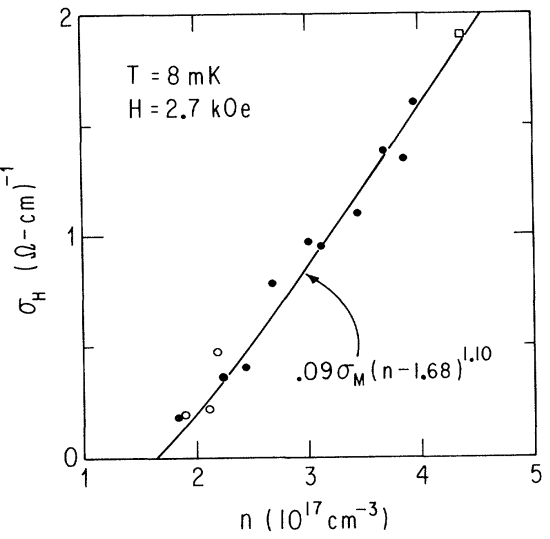

FIG. 2. The Hall conductivity $\sigma_{\mathrm{H}}$ also scales critically at the MI transition, with a best-fit exponent close to that of $\sigma$ in Fig. 1. Scaling theory of localization predicts a ratio of 2 between exponents.

the Mott conductivity $\sigma_{M}$ is taken to be $7(\Omega-\mathrm{cm})^{-1}$. We compare these results to measurements of the Hall conductivity $\sigma_{\mathrm{H}}$ at the same $T$ and $H$ in Fig. 2. We find scaling behavior with exponent $\nu_{\mathrm{H}}=1.1 \pm 0.2$. Here, the solid line is a two-parameter nonlinear least-squares fit with $\sigma_{1}=0.09 \sigma_{\mathrm{M}}, \nu_{\mathrm{H}}=1.10$, and $n_{c}$ constrained to be $1.68 \times 10^{17} \mathrm{~cm}^{-3}$, as determined from the fit to the longitudinal conductivity.

Measurements of $\sigma(n)$ and $\sigma_{\mathrm{H}}(n)$ at a slightly higher magnetic field yield consistent results. At $H=5.4 \mathrm{kOe}$ the best fit values are $\nu=0.98$ and $\nu_{\mathrm{H}}=1.24$. The ratio $\nu_{\mathrm{H}} / \nu$ remains the same for both fields, $1.2 \pm 0.2$, in contrast to the scaling theory of localization prediction that $\nu_{\mathrm{H}} / \nu=2$. We believe that these measurements are in the appropriate $H \rightarrow 0 \mathrm{lim}-$ it. They are taken in the low-field regime where both the Hall resistivity $\rho_{\mathrm{H}} \propto H$ (see the inset to Fig. 3) and the longitudinal magnetoresistivity $\rho \propto H^{2}$. In addition, $\nu$ has not changed from its $H=0$ value of $0.9 \pm 0.1$.

The scaling theory of localization predicts a drop in the conductivity at the approach to the MI transition over and above the more gradual decrease expected from the decreasing number of carriers. In particular, Shapiro and Abrahams predict that the Hall coefficient $R_{\mathrm{H}}$ should remain constant as $n \rightarrow n_{c}$. We plot $1 / R_{\mathrm{H}}$ vs $n$ at $T=8 \mathrm{mK}$ in Fig. 3. The values of $R_{\mathrm{H}}$ $=d \rho_{\mathrm{H}} / d H$ were determined from linear fits to the low-field behavior of $\rho_{\mathrm{H}}$, shown for a representative sample in the inset to Fig. 3. The Hall coefficient data clearly go to zero as $n \rightarrow n_{c}$, with an exponent $\nu_{\mathrm{RH}}$ $=0.7 \pm 0.15$. The value of $\nu_{\mathrm{RH}}$ follows directly from $\nu$ and $\nu_{\mathrm{H}}$ via the matrix relation $\rho_{\mathrm{H}}=\sigma_{\mathrm{H}} /\left(\sigma^{2}+\sigma_{\mathrm{H}}^{2}\right)$. Preliminary results ${ }^{11}$ on the magnetic semiconductor $n$-type EuTe also indicate that $R_{\mathrm{H}}$ changes with $n$ near $n_{c}$.

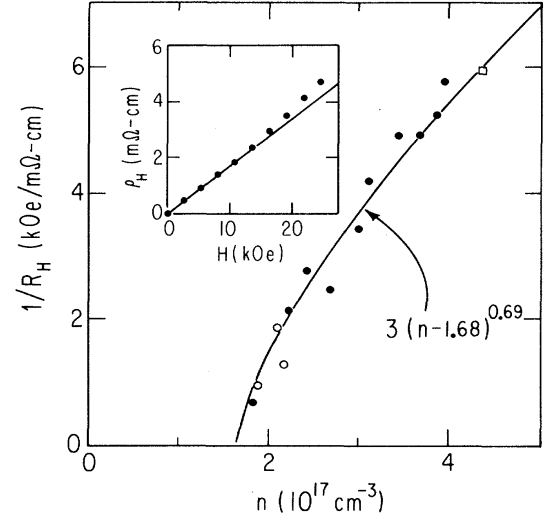

FIG. 3. Critical behavior of the inverse of the Hall coefficient $R_{\mathrm{H}}$. Theory predicts no dependence on $n$. We determine $R_{\mathrm{H}}$ from the slope of the Hall resistivity $\rho_{\mathrm{H}}$ vs magnetic field $H$ in the linear regime, as plotted in the inset for $n=3.95 \times 10^{17} \mathrm{~cm}^{-3}$ sample.

Our results on $\mathrm{Ge}: \mathrm{Sb}$ disagree with the predictions of the scaling theory of localization as extended to include the critical behavior of the Hall conductivity. It may be that the measurements reported here are not close enough to $n_{c}$, although previous experiments ${ }^{2,4}$ on $\mathrm{Si}: \mathrm{P}$ and $\mathrm{Ge}: \mathrm{Sb}$, as well as a calculation by Bhatt and Ramakrishnan, ${ }^{12}$ suggest that we are within the critical region. It is also possible that a small but finite magnetic field has changed the universality of the transition. It seems more likely that a one-electron viewpoint is just not adequate, even for disordered systems where $\nu=1$. We note that the high-field magnetoresistance of these Ge:Sb samples is positive and proportional to $H^{1 / 2}$, indicating a contribution from electron interaction effects. ${ }^{13}$ It would not be surprising to find that the interactions also drive the critical behavior, ${ }^{6,7}$ but we await explicit calculations of $\nu_{\mathrm{H}}$ before we can make a more definitive statement.

We would like to thank H. Fritzsche both for supplying the samples and his active interest and advice. We have benefitted from discussions with E. Abrahams and P. A. Lee. This work was supported by the National Science Foundation under Grant No. DMR 83-05065. One of us (T.F.R.) acknowledges receipt of an Alfred P. Sloan fellowship.

${ }^{1}$ E. Abrahams, P. W. Anderson, D. C. Licciardello, and T. V. Ramakrishnan, Phys. Rev. Lett. 42, 693 (1979).

${ }^{2}$ T. F. Rosenbaum, K. Andres, G. A. Thomas, and R. N. Bhatt, Phys. Rev. Lett. 45, 1723 (1980); M. A. Paalanen, T. F. Rosenbaum, G. A. Thomas, and R. N. Bhatt, Phys. Rev. Lett. 48, 1284 (1982).

${ }^{3}$ G. Hertel, D. J. Bishop, E. G. Spencer, J. M. Rowell, and 
R. C. Dynes, Phys. Rev. Lett. 50, 743 (1983).

${ }^{4}$ G. A. Thomas, Y. Ootuka, S. Katsumoto, S. Kobayashi, and W. Sasaki, Phys. Rev. B 25, 4288 (1982).

${ }^{5}$ S. von Molnar, A. Briggs, J. Flouquet, and G. Remenyi, Phys. Rev. Lett. 51, 706 (1983).

${ }^{6}$ C. Castellani, C. Di Castro, P. A. Lee, and M. Ma, Phys. Rev. B 30, 527 (1984).

${ }^{7}$ A. M. Finkelstein, Zh. Eksp. Teor. Fiz. 84, 168 (1983) [Sov. Phys. JETP 57, 97 (1983)].

${ }^{8}$ B. Shapiro and E. Abrahams, Phys. Rev. B 24, 4025
(1981).

${ }^{9}$ T. F. Rosenbaum, K. Andres, and G. A. Thomas, Solid State Commun. 35, 663 (1980).

${ }^{10} \mathrm{H}$. Fritzsche, private communication.

$11 \mathrm{~J}$. Stankiewicz, S. von Molnar, and F. Holtzberg, to be published.

12R. N. Bhatt and T. V. Ramakrishnan, Phys. Rev. B 28, 6091 (1983).

13P. A. Lee and T. V. Ramakrishnan, Phys. Rev. B 26, 4009 (1982). 